\title{
The Bonding of Gold and Gold Alloys to Non-Metallic Materials
}

\author{
William S. Rapson \\ International Gold Corporation Limited, Johannesburg, South Africa
}

\begin{abstract}
A number of industrial applications of gold are based upon the bonding of it or its alloys to non-metallic substrates. Such applications are reviewed in this article with special reference to the techniques employed and the bonding mechanisms which are involved.
\end{abstract}

The fact that gold and gold alloys can be made to adhere to a variety of non-metallic substrates is responsible for a number of their applications. Perhaps the oldest of these are the bonding of gold to glass, which is an integral step in the production of gold mosaics, and its bonding to ceramic substrates which has long been exploited in the decoration of ceramic-ware and in the enamelling of carat golds in jewellery. Although applications such as these developed initially as crafts, they have acquired a more scientific and technical basis in recent years. At the same time the bonding of gold and gold alloys to these and other non-metallic substrates has been exploited in a number of different contexts. It is now applied for example in dentistry where porcelain veneers on gold alloy restorations are widely used and, in the form of leaf or foil, gold can also be used as an interlayer to facilitate bonding between nonmetallic materials.

The most sophisticated applications of gold, for which its adhesion to non-metallic materials is of importance, have been developed, however, in microelectronics. In thick film metallization processes, conductive gold and gold alloy patterns are applied to oxide substrates by techniques which have evolved directly from those first used in the ceramics industry. In thin film processes, on the other hand, gold metallization is applied by vacuum techniques either to bulk oxides such as alumina, to thick oxide insulating films, or to thin naturally occurring oxide layers on metallic or semi-metallic substrates.

In all these applications the strength with which the gold adheres to the substrate is of great importance. The bonding mechanisms and the factors which affect the adhesion of gold to such substrates are therefore of considerable significance.

\section{Bonding of Gold Alloys to Dental Porcelains}

Although in the jewellery industry there is a long experience $(1,2)$ of the enamelling of a wide range of carat gold alloys, little of a scientific or technical nature appears to have been reported in regard to it. This is in strong contrast to the position in respect of the bonding of dental gold alloys to dental porcelains illustrated by Figure 1 .

Apart from bonding firmly with the dental porcelain on firing, dental gold alloys to be used with enamel veneers in dental restoration must meet other stringent requirements. They must not discolour the porcelain on firing, their coefficient of thermal expansion must be such as to ensure that cracking and spalling of the enamel do not take place on cooling and

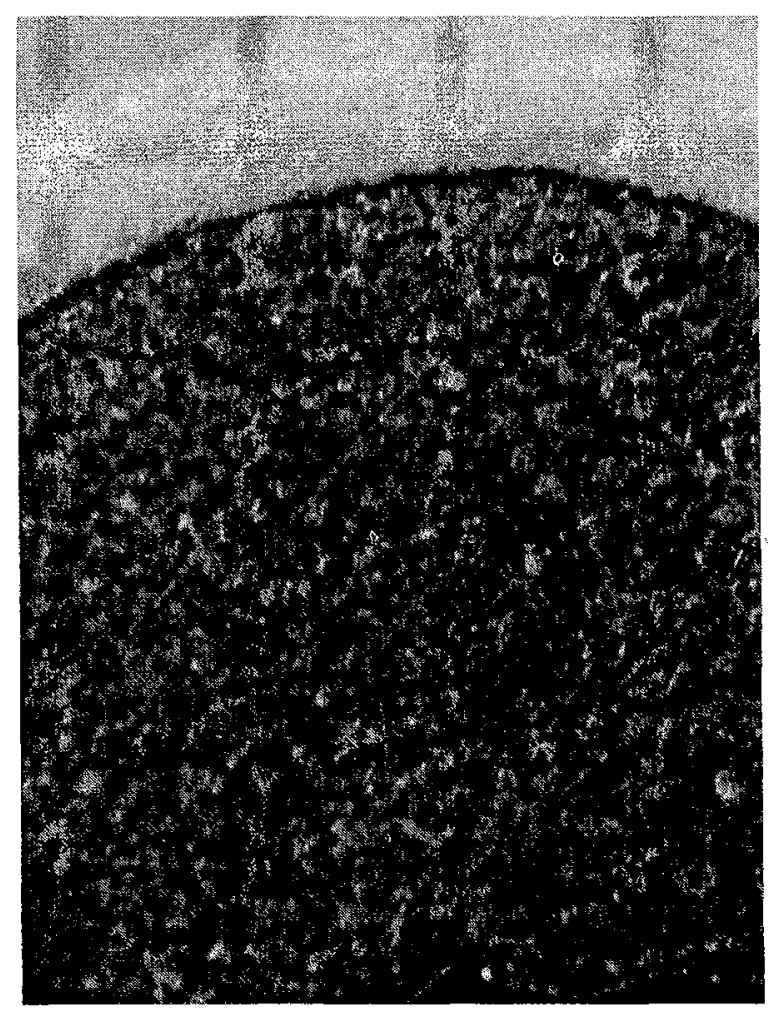

Fig. 1 Cross-section of part of a gold alloy dental restoration with a porcelain veneer. Gold alloys formulated for this type of application must have excellent mechanical properties, bondability, controlled thermal expansion and should not contaminate the ceramic or alter its colour Sample by courtesy of Argen (Pty.) Ltd., Edenvale, South Africa 
they must be hard and strong enough to provide rigid support for the brittle enamel when under stress.

The alloys found most suitable are essentially goldpalladium-platinum alloys to which iron, indium and tin are added in amounts of up to 1 per cent along with grain refining agents. The iron, indium and tin act as age hardening agents, and together with small amounts (less than 1 per cent) of silver and copper which are usually added, they also promote what is known as reaction bonding of the metal to the porcelain. Additions of other elements such as aluminium (3), silicon (3), zinc (4) or titanium (5) may also be made for this purpose.

Sperner (6) has reviewed the mechanisms by which this reaction bonding takes place between alloy and porcelain. Although mechanical interlocking and ease of wetting may play a part, the overriding importance of oxide layers on the surface of the metal phase was early recognised. The fundamental observations were made, however, in respect of the enamelling of steel by King, Tripp and Duckworth (7), who concluded in 1959 that during firing there was a continuous exchange of an oxidation-reduction type between metal ions in the ceramic and metal atoms in the alloy.

Experimental evidence for the occurrence of such an interchange in the bonding of dental gold alloys to dental porcelains has recently been provided by Sperner (6). Using microprobe analysis, he was able to demonstrate (see Figure 2) not only the migration of easily oxidizable metals such as indium and tin from the alloy to the ceramic phase on firing, but also the movement of silicon, aluminium and oxygen across the interface from the ceramic to the alloy.

The work of the many other investigators who have studied different aspects of such reaction bonding, and in particular the work of Lautenschlager et al. (8), von Radnoth (9) and Nally et al. (10) must be read against the background of these observations.

\section{Bonding in Liquid Gold and Thick Gold Film Technologies}

Although gold has long been used in the form of powder for the decoration of ceramics, the films obtained were thick (about $25 \mu \mathrm{m}$ ) and it was only with the development of liquid golds in the period 1800 to 1830 and the publication in 1851 of details of their preparation and use that the formation of thinner, bright and less costly gold films on ceramics became possible.

Liquid golds are basically solutions in an organic medium of thermolabile gold mercaptides of the structure Au-S- $\underline{R}$, where $\underline{R}$ may be either a terpenyl or an alkyl (for example, a tertiary dodecyl) group. When such solutions are applied to ceramic substrates and fired, the organic matter and sulphur are volatilized or oxidized away and an adherent film of metallic gold is left behind.

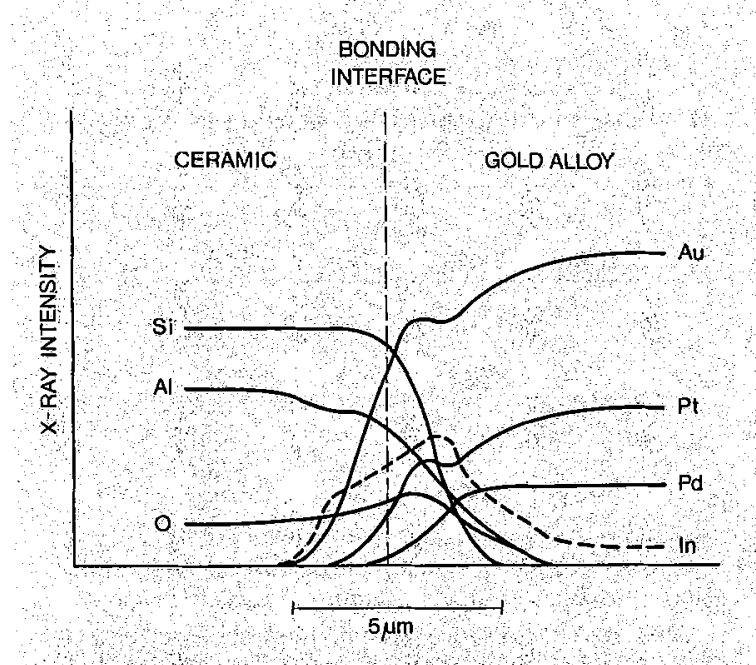

Fig. 2 Schematic concentration profiles in the vicinity of the interface between Herador $\mathrm{H}$, a dental gold alloy of W, C. Heraeus GmbH, and a ceramic after firing in air for 5 minutes at $950^{\circ} \mathrm{C}$. The profiles were determined with an $X$-ray microprobe. Note the indium enrichment at the interface, After (6)

It is highly significant, however, that it was early (11) found necessary to incorporate into such solutions not only resins and related materials in order to adjust their viscosity and other properties to the needs of brushing, spraying, screen-printing etc., but also soluble salts of a variety of elements such as bismuth, lead, tin, nickel, chromium, vanadium, iron, silicon and rhodium. These decompose on firing to leave a residue of their oxides dispersed in the gold. As in the case of the bonding of dental gold alloys to dental porcelain, the presence of the base metal oxides is necessary in order to promote reaction bonding and ensure strong adhesion of the metallic gold to the ceramic subtrate. The addition of rhodium brings an additional benefit since it has been found effective in preventing agglomeration of the gold by promoting wetting and the formation of a smooth and lustrous gold film (12).

In another technology, namely that of microelectronics, there is now extensive experience of a similar need for the presence of base metal oxides in order to make gold adhere strongly to alumina substrates.

Thick film technology is based upon the use of thick film pastes. In these, gold powders, either alone or in admixture with powders of silver, platinum and palladium, are blended in desired proportions with an organic vehicle and a bonding agent. The organic vehicle must be such as to facilitate the screenprinting of the paste onto alumina substrates. On subsequent firing, the printed paste patterns generate the extremely fine gold or gold alloy circuits required.

Two types of paste $(13,14,15)$ have been developed which differ in the nature of the agent used to bond the gold to the alumina substrate on firing. 


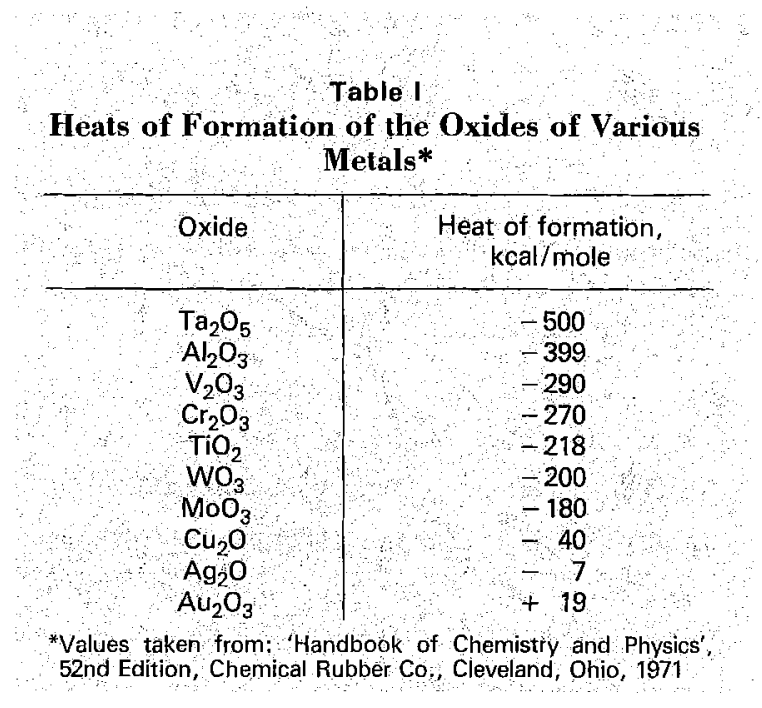

The first type to be manufactured contains glass frit as the bonding agent. On firing, the frit softens and reacts with the ceramic surface. Further glass becomes attached to this anchored glass surface, so that on cooling the gold tends to be largely at the surface of a glass layer to which it is bonded both by mechanical interlocking and by adhesive forces. The use of vanadium oxide (16) and of bismuth oxide (17) in pastes of this type has been claimed to improve the bonding and other properties of the final films.

The second type of thick paste to be developed does not contain glass frit and is based essentially upon application of the experience of reaction bonding of gold in dentistry, which has been discussed above. Reaction bonding of the gold to the alumina substrate on firing is promoted by incorporating in the paste small quantities of base metal oxides, particularly oxides of copper and cadmium (15).

\section{Bonding of Gold in Thin Film Technology}

As mentioned above, the physical deposition processes of evaporation, sputtering and ion plating are extensively used in the production of gold films. It is general experience that when applied to oxide substrates by thin film techniques, only those metals adhere strongly that have a strong affinity for oxygen as reflected by the heats of formation of their oxides. Gold (see Table I) is not such a metal and suffers from an inherent disadvantage in these applications. In microelectronics, thin films of gold are not normally applied directly to oxide substrates by physical deposition processes. Instead, the surface of the oxide is first coated with a metal with a high affinity for oxygen (tantalum, aluminium, vanadium, chromium, titanium, tungsten or molybdenum - see Table I). Subsequently the gold is applied to this 'bonding metal' film.
It is for this reason that a variety of systems is used for the metallization of oxides in microelectronic devices. Typical such systems are tabulated on page 99 of this issue of Gold Bulletin.

A recent observation of interest is that strong adhesion of vacuum-evaporated gold to alumina, silica and glass surfaces is achieved by the interposition of films of cadmium, tin, lead, indium or bismuth fluorides (18). The fluorides etch and bond to the substrate surfaces and the gold is described as bonding to the fluoride surfaces because of its ability to 'alloy' with the metals of the deposited fluorides without destroying the latter. Another observation is that silicon adheres strongly to gold electroplated from a bath containing a tantalum complex, even though the electrodeposit contains less than $10 \mathrm{ppm}$ of tantalum (19).

The factors affecting the adhesion of thin films of metals to oxides have been reviewed by Mattox (20) who has discussed the importance of the structure of the interfacial region in determining this adhesion, as well as the ways in which this interfacial structure may be affected not only by nucleation, film growth and surface effects, but also by the conditions under which the metal film is applied.

There are basic differences between the adhesion of metals applied by vacuum processes and those applied by other methods. In vacuum processes, for example, gold is not brought into contact with the substrate in bulk or necessarily at a high temperature. It arrives at the surface in the form of atoms or ions and thin film formation occurs as a result of crystal growth in the plane of the surface. The process is not greatly different from that involved in electrodeposition of gold coatings. Moreover, just like electrodeposited gold films, thin gold films may possess properties which differ from those of gold in bulk. For example, their fracture properties, which affect their adhesion, may be different.

Surface diffusion of the metal on the substrate to sites suitable for crystal growth plays an impontant role in the formation of thin films. If mobility is high as in the case of gold on carbon, nucleation density tends to be low and the nuclei may grow vertically as well as laterally to form island-channel structures which may reach appreciable thickness before they are 'bridged' and the film becomes continuous. This is typical of a 'non-wetting' type of growth, in which (Figure 3) a lack of overall contact and the presence of voids at the interface even when the film is continuous may lead to easy fracture propagation in the interfacial region and low film adhesion. In some cases of this kind, particularly at elevated temperatures, the nuclei may tend to 'de-wet' from the surface, in which case these effects may be enhanced. In contrast, where surface mobility is low or the substrate contains many local defects or impurity atoms, the nucleation density tends to be high 
and the film becomes continuous at relatively low thicknesses. This is the case when aluminium is deposited. This metal has low mobility on oxide surfaces because it reacts strongly with them. It displays a 'wetting type of growth (Figure 3) which is highly favourable to strong adhesion.

Apart from the effects of diffusion and nucleation rates, the later stages of thin film formation may be af fected also by the mobility of the nuclei and their ability to coalesce. Temperature effects, epitaxial growth and charge effects may all play a role in this connection.

The extent and the nature of the interaction between the atoms of the plating metal and the nonmetallic substrate are also greatly affected by the energies with which the atoms arrive at the surface. In vacuum evaporation, the metal to be deposited is vaporized and condensed on the substrate surface in a vacuum which is high enough to limit collisions between metal atoms and residual gas over the distance between the source of evaporationg metal and the substrate. Only thermal energies $(0.1$ to $0.5 \mathrm{eV})$ and line of sight deposition are involved. In sputtering, the metal atoms are ejected from the source as a result of impact with high energy particles. They in turn strike the substrate with greater energy (1 to $100 \mathrm{eV})$. Their high condensation energy, their ability to clean the substrate surface and to create local defects on it all contribute towards making sputtered films more adherent than evaporated ones. In ion plating the particle energies involved are still higher (21) and the films formed are correspondingly more adherent.

In the case of thin films of reactive metals on oxides, the reaction zones, like those between dental gold alloys and dental porcelains, typically consist of complex oxide phases involving both the metal and the substrate materials. They may be 10 to $100 \AA$ thick but greater thicknesses may adversely affect adhesion because of high stress gradients between the phases formed.

In the case of a noble metal such as pure gold the reaction zones are absent and a sharp compositional discontinuity from one material to another is always observed.

\section{Bonding of Gold and Gold Alloys to Glasses}

In the early days of mosaic art, the gilding of the glass tesserae was carried out by the cold application of fine gold leaf to the glass, which was covered with powdered glass and fired (22). In present day practice, pure gold leaf is applied in squares $(10 \times 10 \mathrm{~cm})$ to the surface of a circular damp glass sheet ( $1 \mathrm{~mm}$ thick) over which molten glass of the required colour is poured. The material is then fired at around $1200^{\circ} \mathrm{C}$, annealed and cut into strips from which the artist cuts tesserae of the size required.

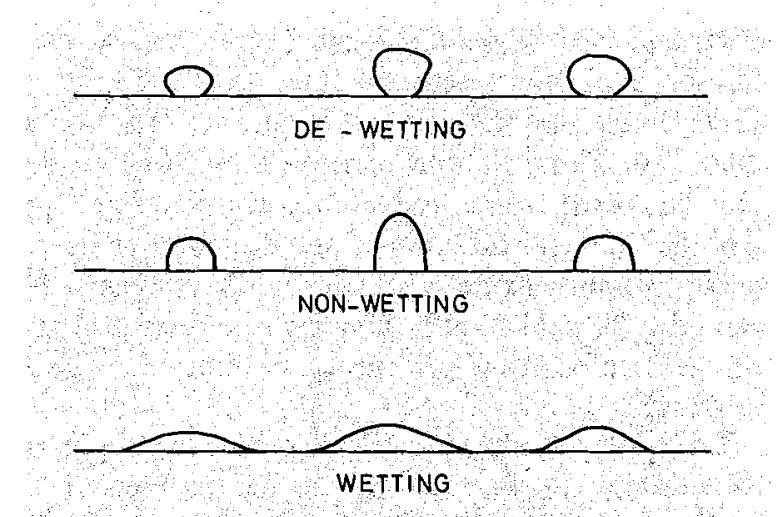

Fig, 3 Schematic representation of de-wetting, non-wetting' and wetting nucleation and growth in the deposition of a metallic film on a non-metallic surface, Non-wetting nucleation and growth are exhibited by gold vacuum-deposited onto carbon. After (20)

Similar bonding of gold to glass has been exploited in the fabrication of vacuum tubes. The leads to these are normally of nickel-chromium-iron or iron-cobaltnickel alloys, whose thermal expansion coefficients match that of the glass. These alloys have electrical resistivities which are great enough to cause local heating of the glass-to-alloy seal and this is overcome by applying a gold coating ( 25 to $30 \mu \mathrm{m}$ thick) to the alloy before sealing.

More recently, the bonding of gold to glass has become important in the production of reflector and absorber coatings on glass by thin film techniques and in the use of frit bonding thick film gold pastes in microelectronics.

Pure gold is not highly adherent to ordinary glass when applied to it by vacuum tecniques. The interface between the metal and the glass is sharp and there is no apparent reaction zone. Adhesion can be greatly increased, however, by pre-treatment of the glass surface. For example, the preliminary applica:tion to the glass surface of a very thin film of nonstoichiometric bismuth trioxide (23) greatly increases the nucleation density and therefore the adhesion of the gold. Alternatively, the glass may be precoated with very thin films of aluminium, tin or cerium oxides. Such techniques are used in the application of heat reflective gold films to glass intended for the insulation of buildings from solar heat (24). They are also used in the coating with electrically conductive gold films of the glass windows of vehicles or aircraft operated under misting or icing conditions. The ability of interposed films of certain metallic fluorides to promote adhesion of pure gold to glass has been referred to above (18).

If in the place of pure gold, gold alloys containing base metals are used, or if in place of ordinary glasses high lead glasses are used, then much more adherent 
films may be obtained. Gold adheres well to high lead glasses, probably because of interfacial diffusion of lead cations from the glass and of gold atoms from the gold (20). Thus, alumina substrates for gold plating may be coated with a lead aluminosilicate glaze (18). The fact that the addition of small quantities of tin oxide, bismuth oxide or cerium oxide increases the solubility of gold in glass may well be related to the role of such oxides in promoting the adhesion of gold to glass.

The effects of additions of base metals to gold upon its adhesion to glass do not appear to have been studied in detail. In view of the role of base metals in the bonding of dental gold alloys to dental porcelains, such additions should promote bonding. They are almost certainly a factor in the application of enamels to carat golds in jewellery fabrication, where participation of base metals in the process may be evidenced by colour effects in the enamel phase.

More specific evidence of the manner in which additions of base metals to gold may promote its bonding to oxides or ceramic substrates is available from the work of Schmidt-Brücken and Schlapp (25) who have demonstrated that additions of small quantities of metals such as cerium, chromium, gallium, silicon, vanadium and lithium to gold strongly promote its bonding to ceramics.

\section{Bonding of Gold to Non-Metals without Additions of Base Metal Oxides}

Although the effects of base metal oxides in promoting reaction bonding between gold and nonmetallic substrates are now well recognised and although these effects are exploited in a range of different technologies, investigations over the past few years $(26,27,28)$ by the Division of Chemical Physics of the Commonwealth Scientific and Industrial Research Organisation, in Australia, have demonstrated that base metal oxides are not essential to the establishment of strong bonds between gold (and other noble metals) and ceramic substrates. Although the techniques involved have been protected by patents, the nature of the bonding reactions are still under active investigation.

In practice, the components (metal and ceramic) are simply pressed into contact and heated to a high temperature for a predetermined period. The surface of the ceramic is normally flattened and polished by optical grinding techniques. If the metal surface is massive, it is prepared similarly, but if it is a foil which conforms to the surface of the ceramic when pressed, no pre-treatment is required. The pressure applied during the heating cycle is not high, being only sufficient to ensure intimate contact between the mating surfaces. Firing is usually at a temperature which is about nine tenths of the melting temperature of the metal, most of the ceramics used to date having had higher melting points than the metals being bonded to them. The bonds are normally formed in air and inert gas environments have been used only in cases where the metals oxidize in air at the firing temperatures. In no case has it been found necessary to use reducing atmospheres in order to promote effective bonding.

The process can be used with metals and ceramics of varying purity. If the thermal expansions of the metal and ceramics are not matched, however, the bonds may fracture during or after cooling to room temperature. Suitable geometry or appropriate heating and cooling programmes can overcome this problem.

Experience has shown that almost all metals will bond to almost all ceramics using the technique but that there is a difference between the reaction between noble metals and ceramics or oxide crystals on the one hand, and that between other transition metals and such substrates on the other hand.

Whereas the migration of transition base metals into the non-metallic partner can be followed by microprobe over distances of some thousands of Ångströms, the corresponding migration distances for the noble metals are not measurable by electron microprobe and are of the order of tens of Ångströms. Whether this constitutes a difference of degree or kind is not known. The work of J. A. Pask and his coworkers (29) at the Sandia Laboratories in Albuquerque may be significant in this connection. Mechanical interlocking resulting from diffusion of metal into pores of the ceramic is not the basic mechanism, since equally strong bonds can be obtained between a metal and single crystal rather than granular oxides. For example, gold bonds equally strongly to granular alumina and to optically polished sapphire (pure $\mathrm{Al}_{2} \mathrm{O}_{3}$ crystal).

Applications of bonding of this type are being actively pursued. One of the most interesting is in the fabrication of gas-sensing probes, for monitoring the oxygen levels in various high temperature environments such as those prevailing in furnaces in the steel industry, in air-fuel mixtures, in smoke stacks and in car exhausts.

Solid state oxygen-sensing probes are generally composed of one form or another of cubic-stabilized zirconia $\left(\mathrm{ZrO}_{2} / 15\right.$ per cent $\mathrm{CaO}$, for example) which is used as a solid electrolyte. There is easy and rapid diffusion of oxygen ions through such an electrolyte. If, therefore, there is a difference between the oxygen pressures on the two sides of a stabilized zirconia electrolyte, at a temperature in excess of about $500^{\circ} \mathrm{C}$ a difference in non-stoichiometry is established. Electrodes attached to the two sides will then record a potential difference which is a measure of the oxygen pressure ratio between the two exposed zirconia surfaces. 
Fig. 4 Components of a dual gas-sensing solid electrolyte probe, constructed using reaction-bonding techniques. The tube to the right has a $\beta$-alumina wafer at its tip and senses sulphur activity. The $\beta$-alumina wafer (at the bottom of the tube) is bonded via two alumina-gold sections to mullite (the long dark section of the tube), which in turn is connected to the upper glass part. The tube to the left consists of a zirconia thimble (at the bottom of the tube) with a gold electrode bonded to. it at the tip; the bond is completed by an alumina ring. The thimble is bonded to an alumina tube via gold; the alumina tube is bonded to mullite via gold and the mullite is bonded to glass. The tube in the centre consists of a zirconia thimble bonded to alumina which is then bonded to mullite, in both cases via gold. The complex sequences of bonds are designed to cope with the mismatches in thermal expansion. After (26) Reproduced by permission of the Division of Chemical Physics, CSIRO, Clayton, Australia

Suitable stabilized zirconia tubes, thimbles and wafers are difficult to fabricate, expensive and develop leaks in service. Where long probes are required, it has been found that these can be fabricated by joining together short $(10 \mathrm{~cm})$ zirconia closed-end tubes to long alumina tube shafts via gold or platinum bonds. The products have given satisfactory service for more than 6 months at about $1250^{\circ} \mathrm{C}$. Similar probes which have been developed for measuring both the oxygen and sulphur activities generated when sulphide ores are heated, have been made using gold as the 'sandwich' metal because it is resistant to sulphur, whereas platinum is not (Figure 4).

The fact that sapphire and ruby can readily be bonded to gold (or platinum) or to stainless steel via gold, points to potential applications for the new technique in other fields. Thus, it might well be used in production of prefabricated ruby or sapphire bearing units for scientific instruments or in jewellery production.

\section{The Bonding of Gold to Organic Polymer Surfaces}

The weak adhesion of gold films to the surface of organic polymers and the ease with which such films can be transferred to the surfaces of other materials, has resulted in gold coated polymers taking the place of gold leaf in many of its traditional applications in printing, embossing etc. These applications have recently been described (30) but the factors affecting adhesion of gold to different organic polymers do not appear to have been studied in any detail. A recent patent application (31), covering the use of 'cluster ion

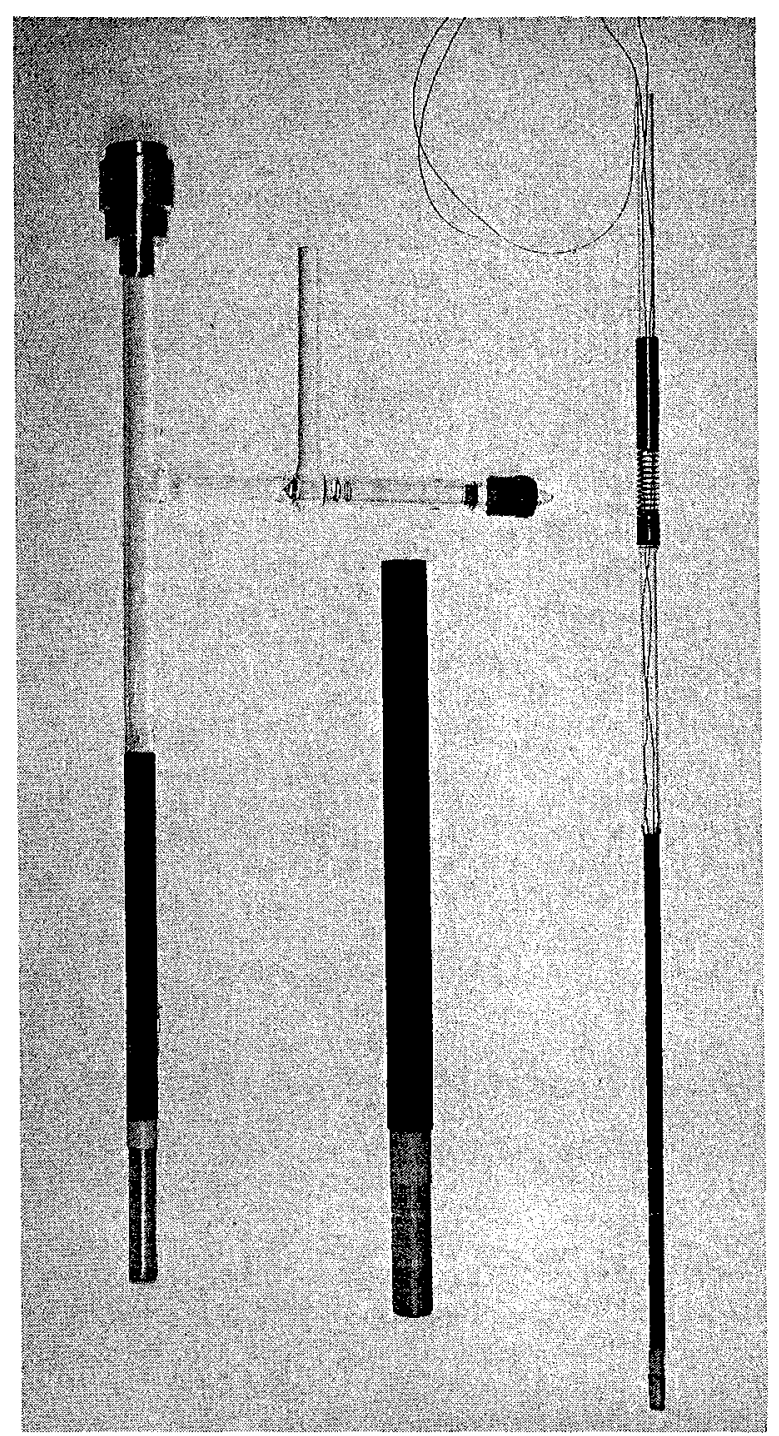

plating' for the application of gold and other metallic coatings to polymer surfaces is of interest in this connection, as is also a claim (32) covering the use of titanium deposited on polyesters to improve their adhesion to gold.

Nenodović et al. (33) have also investigated the mechanical properties, including adhesion, of gold films sputtered onto substrates of acrylate polymers used for the fabrication of artificial teeth and Rogers and Griffith. (34) have discussed the bonding of polycarboxylate cement to gold.

\section{Conclusion}

Because of its low affinity for oxygen, pure gold does not form strong bonds with oxide, ceramic or glass substrates. This has nevertheless not prevented the development of various methods whereby gold can be made to adhere strongly to such substrates in the presence of reactive elements. A number of important applications of gold exploit these techniques. 
In most cases where gold or gold alloys are made to adhere strongly to oxygen-containing substrates, there is evidence of reaction bonding. In some instances, however, where strong bonds are formed between pure gold and these substrates, no evidence of reaction bonding has so far been obtained and the exact nature of the mechanism involved has still to be elucidated.

\section{References}

$1 \mathrm{M}$. Rosenberg, 'Geschichte der Goldschmiedckunst auf technischer Grundlage', Otto Zeller Verlag, Osnabrück, 1972

2 E. A. Smith, 'Working in Precious Metals', N.A.G. Press Ltd., London, 1933

3 Williams Gold Refining Co. Inc., U.S. Patent 3,769,006

4 J. N. Nally, Int. Dent. Assoc. F., 1968, 18, 309-325

5 Dr. Th. Wieland Scheideanstalt, German Offen. 2302837

6 F. Sperner, Z. Metallkd., 1976, 67, 289-295; F. Sperner and N. Harmsen, Mikrochim. Acta, 1977, Suppl. 7, 415-427

7 B. W. King, H. P. Tripp and W. H. Duckworth, f. Am. Ceram. Soc., 1959, 42, 504-525

8 E. P. Lautenschlager et al., 7. Dent. Res., 1969, 48, 1206-1210

9 M. Sz. von Radnoth, Dtsch. zahnärztl. Z., 1970, 25, 259-264

10 J. N. Nally, et al., Schweiz. Monatsschr. Zahnheilk., 1968, 78, 868-878

11 F. Chemnitius, f. Prakt. Chem., 1927, 117, 247-26

12 R. C. Langley, Gold Bull., 1975, 8, (2), 34-40
13 R. G. Finch, Gold Bull., 1972, 5, (2), 26-30

14 R. H. Caley, Gold Bull., 1976, 9, (3), 70-75

15 Anon., Gold Bull., 1975, 8, (1), 13-15

16 E. I. du Pont de Nemours \& Company, U.S. Patent 3,440,182

17 E. I. du Pont de Nemours \& Company, Dutch Applic. 6707540

18 G. J. Zydzik et al., Appl. Phys. Lett., 1977, 31, 697-699

19 S. Serizawa, fapanese Prelim. Publ. 77-129 637

20 D. M. Mattox, Thin Solid Films, 1973, 18, 173-186

21 E. W. Williams, Gold Bull., 1978, 11, (2), 30-34

22 G. Bustacchini, Gold Bull., 1973, 6, (2), $52-56$

23 L. Holland, 'Vacuum Deposition of Thin Films', Chapman \& Hall, London, 1961, p. 504

24 R. Groth and W. Reichelt, Gold Bull., 1974, 7, (3), 62-68

25 H. Schmidt-Brücken and W. Schlapp, Keram. Z., 1971, 23 (4), 191-198; (5), 278-280 and (12), 1-5

26 Annual Report, Division of Chemical Physics, CSIRO, Australia, 1976-1977

27 H. J. de Bruin et al., Gold Bull., 1972, 5, (3), 62-64; C. Varmazis, R. Viswanathan and R. Caton, Rev. Sci. Instrum., 1978, 49, (4), 549-550

28 F. P. Bailey and K. J. T Black, 7. Mater. Sci., 1978, 13, 1045-1052

29 J. A. Pask, Report LBL 602, Sandia Laboratories, Alburquerque, New Mexico, U.S.A., 21 Jan., 1977

30 B. J. Sitch, Gold Bull., 1978, 11, (4), 110-115

31 Sumitomo Bakelite Company Limited and T. Takagi, British Patent 1493088

32 I. Takemoto (Hitachi Ltd.), Japanese Preiim. Publ. 77-136 284 (Chem. Abstr., 1978, 88, 137610)

33 T. Nenodović et al., Thin Solid Films, 1976, 34, 211-214

34 O. W. Rogers and J. R. Griffith, Aust. Dent. F., 1977, 22, (5), $371-372$

\title{
Gold Plating with Pulsed Current
}

\section{PRESENTATIONS AT A RECENT SYMPOSIUM}

\author{
Frank H. Reid
}

Consultant, Werner Flïhmann AG, Duebendorf, Switzerland

\begin{abstract}
At an International Pulse Plating Symposium held under the auspices of the American Electroplaters' Society on April 19th and 20th, 1979, in Boston, MA., a number of papers was presented on the pulse plating of gold. The high promise for improved gold coatings prepared by this method of deposition is reflected by this review of current developments.
\end{abstract}

It is now almost a decade since interest in pulse plating as a technological rather than a pure research tool was stimulated by the work of Avila and Brown (1), who utilized pulses and rest periods in the millisecond range to improve the density and electrical conductivity of gold deposits for conducting paths in electric circuits. In the intervening period numerous papers have been published in this field (for example, the review by Raub and Knödler of the application of the technique to the plating of gold, Gold Bull., $1977,10,(2), 38-44)$ and investigational work has also extended into the plating of base metals, such as chromium, copper, zinc and cadmium.

As for all relatively new techniques, wide claims have been made concerning the benefits to be derived from electroplating with pulsed current ( $\mathrm{PC}$ ), and a principal object of the present Symposium was to provide a clear assessment of the actual 'state of the art', with reference to theory and industrial practicality. In this context, gold plating figured prominently, in view of the promise of more economic usage of the metal via improvements in properties.

Main interest centred on the widely used acid cyanide electrolytes containing cobalt additions. Knödler (2) presented the results of an extensive study of the effects of PC plating on the composition and some mechanical properties of deposits from gold-cobalt, gold-nickel and goldiron electrolytes, with the general conclusion that PC increases the base metal content and decreases the nonmetallic content of deposits, with significant variations of composition and properties possible under varying pulse conditions. Hardness is little affected, but tensile strength is reduced and brightness diminished. The results were interpreted in terms of an adsorption-desorption model on the lines of those proposed by Cheh (3) and Ibl et al. (4). The high hardness of direct current (DC) deposits was attributed to deposition of non-metallic material rather than to the hardening effect of alloying. 